\title{
Quick Recognition and Elimination of an Additional Signal Caused by Deflection of an Integral Roller Flatness Meter
}

\author{
Huaxin YU, ${ }^{1,2) *}$ Tongyuan ZHANG, ${ }^{1)}$ Shuai ZHANG, ${ }^{1)}$ Dongcheng WANG ${ }^{1,2)}$ and Hongmin LIU ${ }^{1,2)}$ \\ 1) National Engineering Research Center for Equipment and Technology of Cold Rolling Strip, Yanshan University, \\ Qinhuangdao, Hebei, 066004 China. \\ 2) State Key Laboratory of Metastable Materials Science and Technology, Yanshan University, Qinhuangdao, Hebei, 066004 \\ China.
}

(Received on January 13, 2021; accepted on June 29, 2021; J-STAGE Advance published date: August 18, 2021)

\begin{abstract}
Compared with the sectional roller flatness meter, the integral roller flatness meter is more suitable for products with higher requirements on surface quality, and thus becomes the trend of the contact-type flatness meter for cold rolling. Due to its structure design, some problems arise. Under the action of the dead weight or an external load, the deflection deformation of the roller changes the stress state of the sensor installed inside it and generates an additional signal. This paper studies the mechanism of the signal and the recognition and elimination method. First, according to the structure of the detection roller and the connection mode of the sensors, the ideal waveform characteristics of the flatness signal are analysed. Through an experiment on the $650 \mathrm{~mm}$ integral roller flatness meter platform, the ideal waveform and actual waveform characteristics are compared, and the influence of the additional signal on the detection signal is analysed. Then, according to the deflection deformation of the roller and its corresponding stress distribution characteristics, the generating mechanism of the additional signal is revealed. Finally, a minimum error method is proposed, which ensures the elimination of the deflection influence on all detection units in real time. During the process of flatness detection, the zigzag feature of the flatness distribution curve is eliminated; thus, during the process of flatness control, the bad influence of the roller deflection is removed. The industrial application shows that the work presented in this paper can obviously improve the products quality.
\end{abstract}

KEY WORDS: flatness meter; integral roller; additional signal; signal recognition; signal elimination.

\section{Introduction}

As an important raw material for high-end products, coldrolled strips are widely used in automobiles and household appliances, electrical devices and electronics, construction and aviation, etc. With the continuous increase in customers' requirements for the quality of cold-rolled strips, the processing equipment and automatic control technology are constantly being innovated. Flatness is an important quality index of cold-rolled strips, ${ }^{1-3)}$ and flatness detection and control are the key technologies for cold-rolled strip. ${ }^{4)}$ Contact-type flatness meters and flatness control systems are the standard technical equipment ${ }^{5,6)}$ and the inevitable choice for the production of high-grade cold-rolled strip product. ${ }^{7)}$ For a long time, flatness meters equipped on high-end strip cold rolling mills were mostly monopolized by several large companies. ${ }^{8,9)}$

\footnotetext{
* Corresponding author: E-mail: 15033537589@163.com
}

In recent years, a large number of researchers explored and studied the principle and equipment of flatness detection. Abdelkhalek, S et al. ${ }^{10)}$ presented a steady state elasticviscoplastic finite element model to the prediction of strip flatness and out of bite buckling during cold rolling of thin strips. Dinh Cuong Tran et al. ${ }^{11)}$ analysed the influence of surface imperfections and global tension on the wrinkling characteristics. Guan, Ben et al. ${ }^{12)}$ presents a novel contactless stress detection technology based on a magnetoresistance sensor and the magnetoelastic effect, enabling the detection of internal stress in manufactured cold-rolled strips. Weisz-Patrault, Daniel ${ }^{13)}$ presented a semi-analytical inverse Cauchy problem for a finite cylinder with a cylindrical hole under the surface called the detecting roll to evaluate the residual stress profile of the strip. Yoshito, Isei et al. ${ }^{14)}$ developed a new shape meter which employed LED dot pattern projection method to realize accurate AFC (Automatic Flatness Control). Shinichiro, Aoe et al. ${ }^{15}$ applied a LIDAR (light detection and ranging) system to 
a plate flatness evaluation system and reconstructed plate flatness surfaces from many points generated by LIDAR by a smoothing spline method. The strip flatness measurement and control technology team of Yanshan University independently developed the integral roller flatness meter ${ }^{16)}$ and a wireless embedded signal transmission and processing device, ${ }^{17}$ which have been used in a series of practical applications in industrial strip cold rolling mills. ${ }^{18)}$ In the process of flatness detection, how to identify and eliminate the influence of additional signals and improve the detection accuracy has always been an important research topic.

Some studies have been carried out on noise elimination and error compensation of the flatness detection signal. Huaxin $\mathrm{Yu}$ et al. $^{19)}$ realized decoupling of the channels of the WRS flatness meter and improved flatness detection and controlling accuracy. By analyzing data processing methods, Jiawei Liu et al. ${ }^{20)}$ applied channel number interpolation and filtering methods to process the detection data, which suppressed the interference influence in the flatness detection data and improved the anti-interference ability of the detection system. Dongcheng Wang et al. ${ }^{21)}$ presented a new method to eliminate the deflection signal using golden section optimization. Haimiao $\mathrm{Wu}$ et $a .^{22-24)}$ analysed the temperature field of the integral detection roller and derived a sensor reliability evaluation model. Yang Lipo et al. ${ }^{25,26)}$ established the cold-rolled strip online dynamic wrapping angle compensation model and a high-precision flatness detection model which consists of wide channels in the middle of a detection roller and narrow channels on both sides to analyse the problem of flatness detection errors. Bingqiang $\mathrm{Yu}$ et al. $^{27)}$ analysed the influences of the measuring error of the strip edges, the transverse temperature difference of the strip, the deflection of the flatness detection roller, and the shape of the strip coil on the flatness measuring results in detail, and established the corresponding compensation models.

In the test calibration and industrial application of the integral roller flatness meter, it has been found that the detection roller units output approximately sinusoidal waveform signals with a $360^{\circ}$ full cycle distribution along the circumference when the roller is rotating and only subjected to gravity. The amplitude of the additional waveform increases when the detection roller is subject to calibration pressure or strip pressure, which will be superimposed with the valid signal and cause flatness detection error. Especially when rolling a thin strip, due to the small tension, the flatness detection error can reach more than $15 \%$. Therefore, it is necessary to study and propose a method to identify and eliminate the waveform to improve the detection accuracy.
In this paper, according to the structural characteristics of the integral roller, it is revealed through mechanical analysis that the additional waveform is caused by the deformation of the roller and the change in the internal sensor stress state. Then, according to the principle of minimum error, a curve fitting method is proposed to identify the waveform, including determination of the amplitude, phase and zero drift. An application example shows that the flatness detection accuracy and the control effect can be significantly improved by eliminating the additional waveforms in all the detection units of the roller.

\section{Integral Detection Roller Structure and Output Waveforms}

Figure 1 shows the structure of the detection roller and the installation and arrangement of the sensors. The number of through holes (usually 2 or 4 ) in which the sensors are mounted depends on the number of peak values of the flatness signals to be tested in one rotation of the detection roller. In the case of a high rolling speed and a small roller diameter, 2 rows of sensors are arranged in the roller body to meet the application requirements. This paper takes the detection roller with 2 rows of sensors as an example to study. Two sensors at the same axial position of the detection roller (circumferential symmetry, $180^{\circ}$ apart) constitute a detection unit, also known as a detection channel. The two sensors in the detection unit output a signal waveform as the roller undergoes one rotation. To facilitate signal extraction, every detection unit is connected to a charge amplifier and a signal processing circuit separately. The two sensors installed symmetrically around the circumference in a detection unit are both composed of two quartz crystals and connected in parallel to improve the sensitivity of the output, as shown in Fig. 2.

In the actual situation of flatness detection, the maximum wrapping angle of the strip to the detection roller is generally less than $60^{\circ}$, and the strip does not cover two rows of sensors simultaneously. Ideally, when one of the sensors in a detection unit is covered by a strip and outputs a peak shape waveform, the signal waveform from another sensor that is not subjected to any force should be a zero line. In other words, the ideal flatness signal should have two peaks distributed in the small phase region with an interval of $180^{\circ}$, similar to a pulse signal, as shown in Fig. 3. The two peaks of the waveform in the figure are the effective segments of the pressure signal, and the peak value represents the pressure exerted on the sensor when the strip passes the roller surface near the sensor. The output signal values

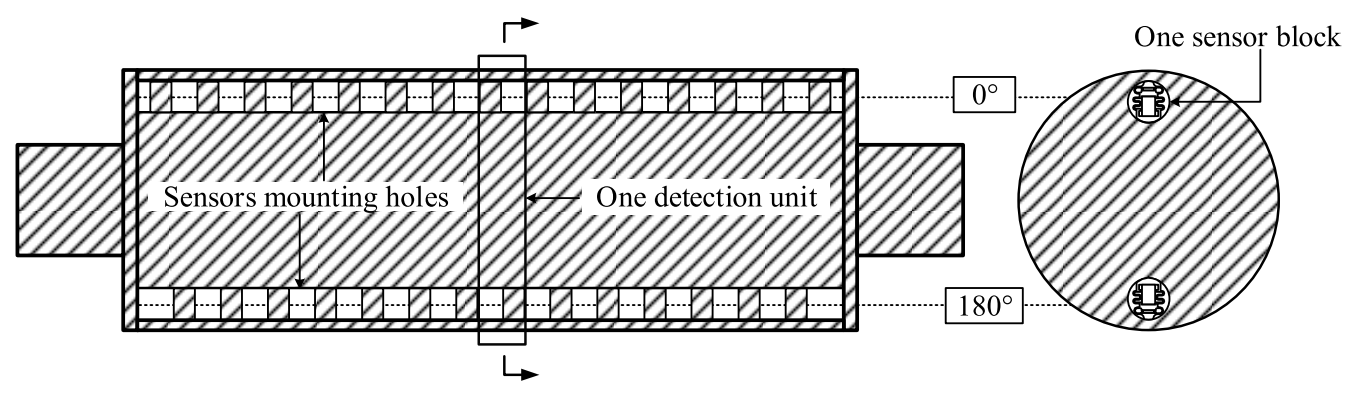

Fig. 1. Structure of the integral detection roller and sensors installation arrangement. 


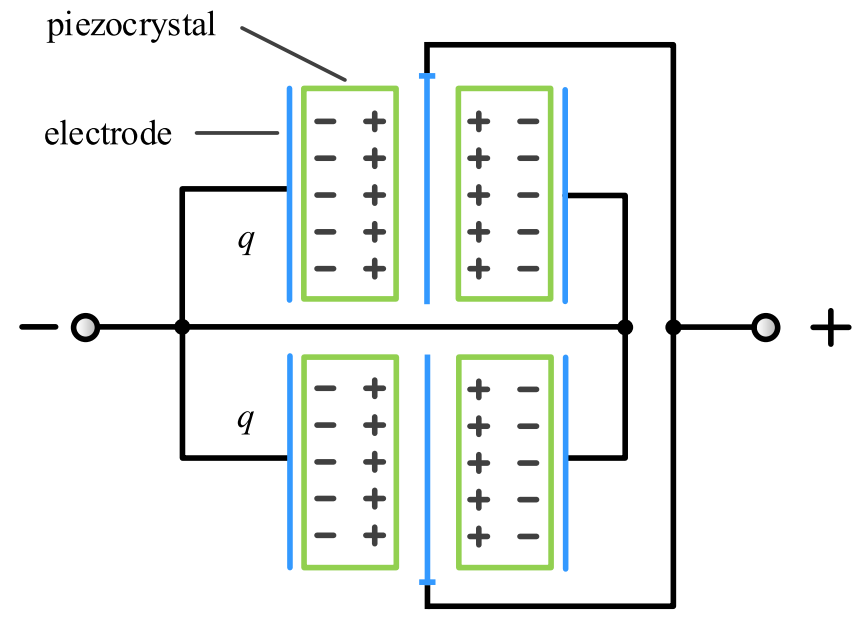

Fig. 2. Parallel connection mode of sensors. (Online version in color.)

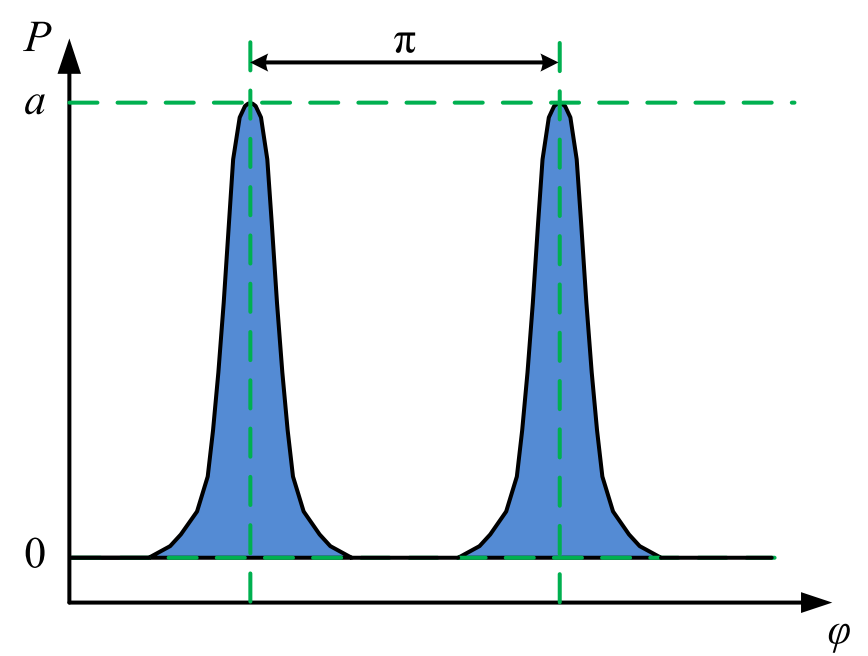

Fig. 3. Ideal waveform. (Online version in color.)

outside the two peak regions should be zero. The amplitude and shape of the two peaks should be exactly the same if the performance of the two sensors is equal and the installation precision is high enough. If different pressures are applied, then the amplitude of the peak will change accordingly. The peak region values should also become zero when the sensors are not under any external load.

To study the original signal output by the integral detection roller, a research platform for the detection roller with a surface length of $650 \mathrm{~mm}$ was built, as shown in Fig. 4. The platform is composed of a detection roller, a driving device, a calibration device, a signal processor (the wireless embedded signal transmission and processing device mentioned above), a high-frequency power supply, a signal receiving computer and a flatness monitor. The platform can test the output characteristics of the sensors under the roller surface. The loading method of the calibration device is shown in Fig. 5. During calibration, the roller body rotates to make the pressure roller rotate in the opposite direction under the action of friction. By means of calibration weights and a calibration rod hinged at one end, the pressure roller exerts a vertical downward radial pressure on a detection unit of the roller. The calibration pressure can be changed by adjusting the weights to regulate the signal strength.

The two sensors (sensor A and sensor B) in a detection

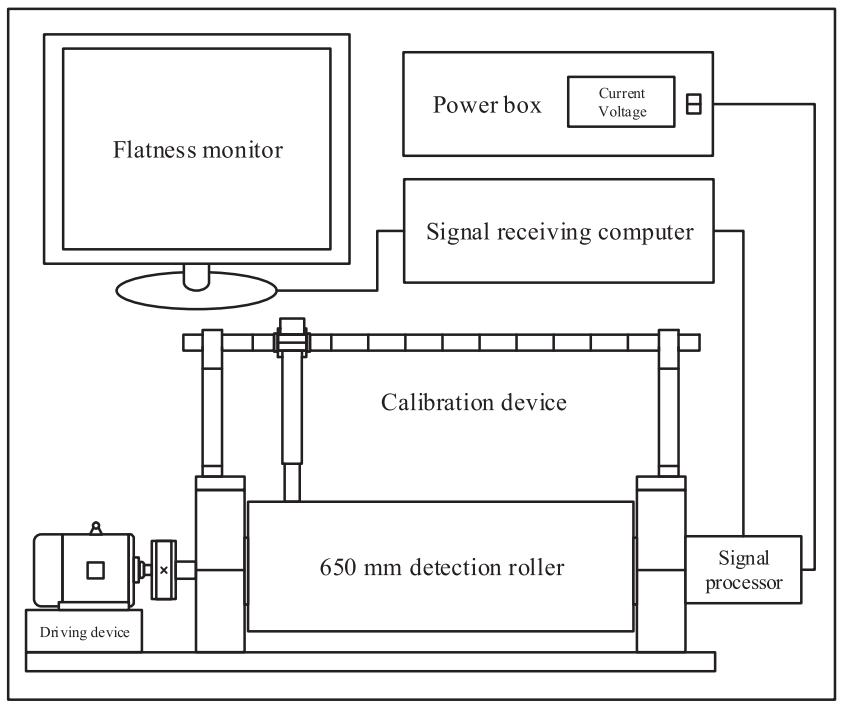

Fig. 4. Experimental platform for the $650 \mathrm{~mm}$ integral detection roller.

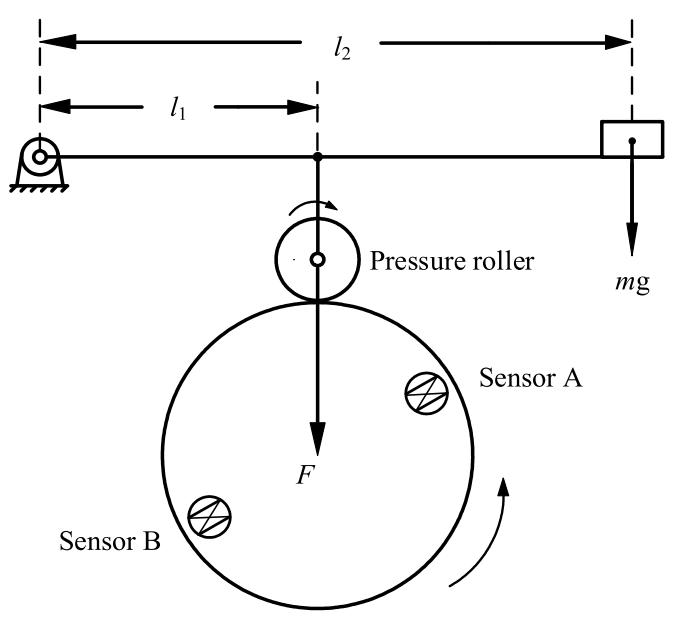

Fig. 5. Loading diagram of the calibration device.

unit are taken as research objects. The two sensors are initially separately connected to the signal acquisition circuit. The output signal waveform when the detection roller idles is shown in Fig. 6(a)). Because the roller is not loaded, the output waveform is supposed to be two zero lines. However, the actual waveforms are two sinusoidal curves with a phase difference of $180^{\circ}$. The amplitude of the sinusoidal curve of sensor A is 280, and that of sensor B is 102 . When sensor A rotates to the bottom and sensor B rotates to the top, sensor A outputs its maximum value, and sensor B outputs its minimum value. When sensor $B$ rotates to the bottom and sensor A rotates to the top, sensor B outputs its maximum value, and sensor $A$ outputs its minimum value.

Sensor A and sensor B are then connected to the signal acquisition circuit in parallel. The waveform when the roller idles is shown in Fig. 6(b)). The amplitude is approximately equal to 280 minus 102 , which is equivalent to the waveform signal obtained after superposition of the two original sinusoidal waveforms, and the phase is the same as that of sensor A.

A radial pressure of $380 \mathrm{~N}$ is applied to the detection unit through the calibration device. When the two sensors are separately connected to the signal acquisition circuit, the 


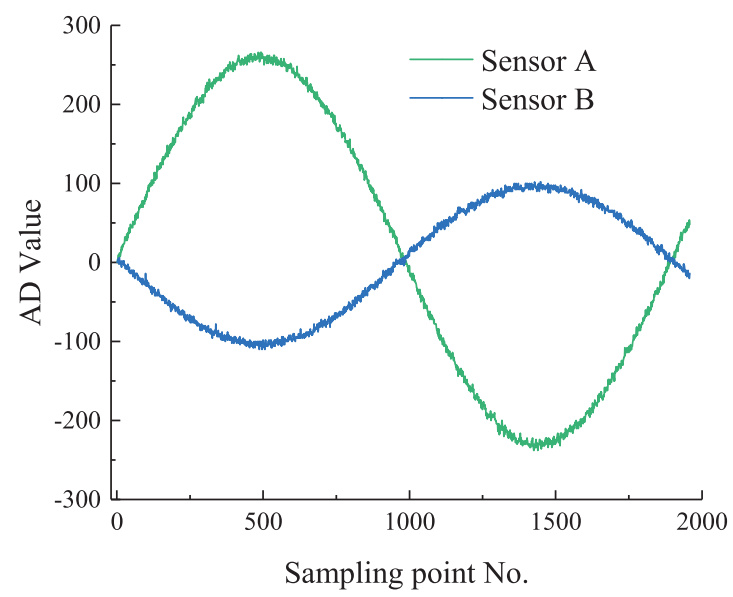

a) Two sensors output separately

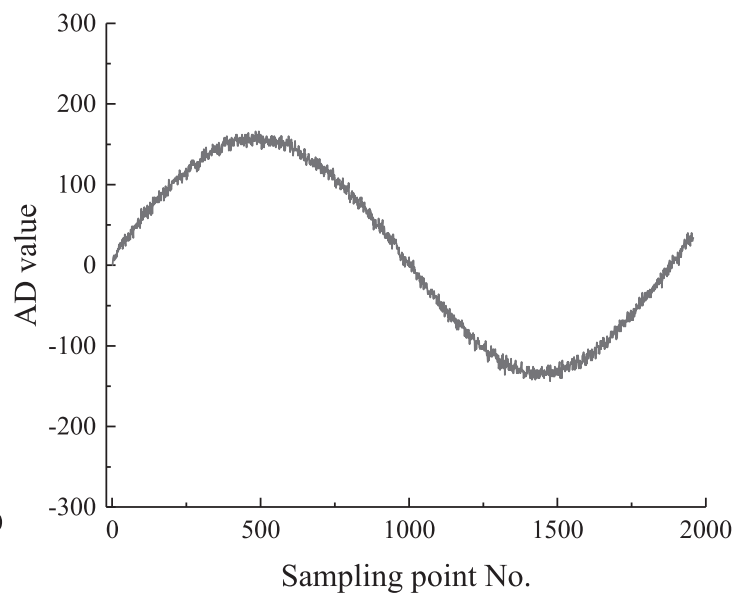

b) Two sensors output in parallel

Fig. 6. Waveforms output when the detection roller is not under load.

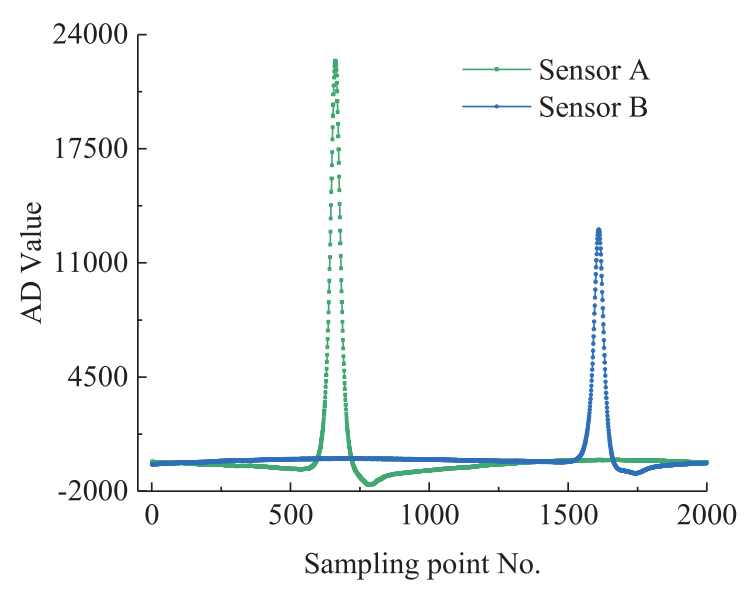

a) Two sensors output separately

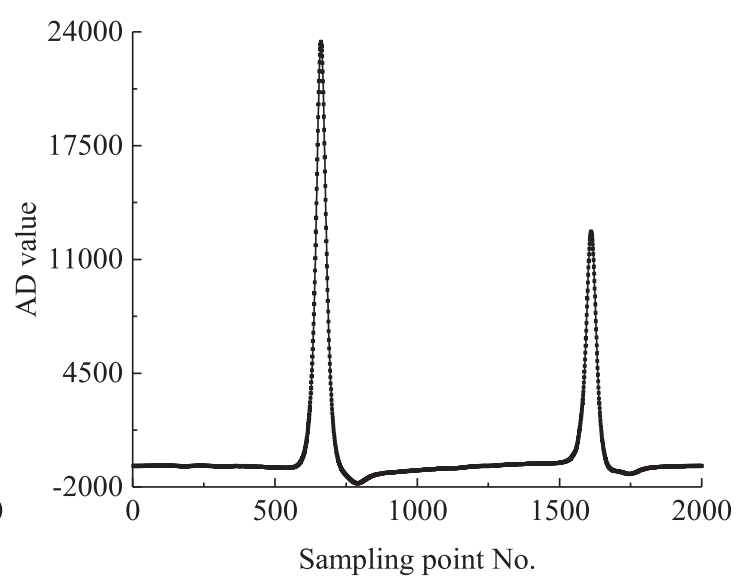

b) Two sensors output in parallel

Fig. 7. Waveforms output when the detection roller is loaded.

output signal waveforms are as shown in Fig. 7(a)). The green curve is the output signal of sensor A, and the blue curve is that of sensor $\mathrm{B}$. The phase difference between them is also $180^{\circ}$.

Sensor A and Sensor B are then connected to the signal acquisition circuit in parallel, and a $380 \mathrm{~N}$ radial pressure is applied to the roller body. The output signal waveform is shown in Fig. 7(b)), which is equivalent to the waveform obtained after the two waveforms are superimposed. By comparing Figs. 6 and 7, it can be seen that the no-load waveform has the same period as the loading waveform, which leads to real-time superposition of the no-load signal and the loaded signal, resulting in a detection error.

By comparing Figs. 3 and 7, it can be seen that the ideal waveform is obviously different from the actual waveform. First, the ideal waveform is very smooth, while the actual waveform has the feature of a "cusp". Second, there is a zero line between the peak regions of the ideal waveform, while the actual waveform has a sinusoidal curve between the peak regions. This indicates that the actual waveform contains interference signals similar to the waveform generated by a variable load, which will definitely affect the flatness detection accuracy.

Take the flatness meter of a $1550 \mathrm{~mm}$ cold rolling mill under no load as an example. The waveform amplitude of different detection units varies along the axial direction. Some detection unit amplitudes are less than 20, which can be almost negligible, while the amplitudes of some detection units can reach 200-300. The mill outlet tension range is $10-120 \mathrm{kN}$. The AD value under the maximum tension is approximately 22000 , and that under the minimum tension is approximately 1820 . At the maximum tension, the influence of the no-load waveform on the detected signal is approximately $1.4 \%$. At the minimum tension, the influence of the no-load waveform on the detected signal exceeds $16 \%$, seriously affecting the detection accuracy.

\section{Mechanism Analysis of the Deflection and Additional Waveform of the Integral Detection Roller}

The sinusoidal waveforms of all detection units distributed along the full cycle of the detection roller are interference waveforms that affect the accuracy of the flatness detection. They are caused by the deformation of the detec- 


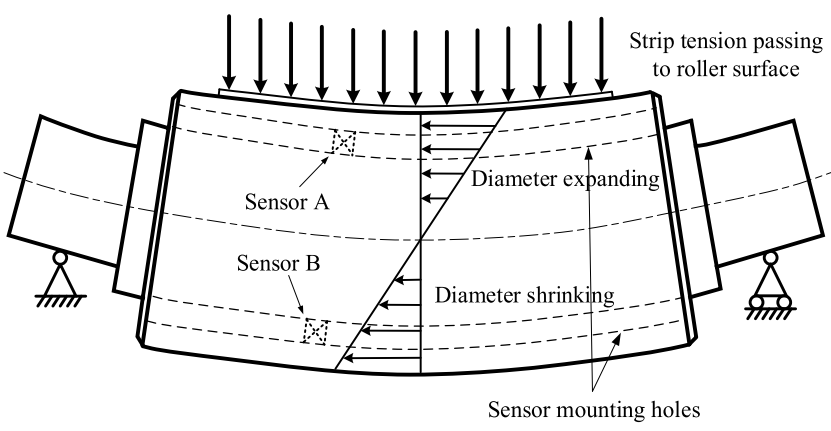

Fig. 8. Enlarged deflection deformation of the detection roller during detecting.

tion roller and the change in the sensor stress state.

As shown in Fig. 8, the detection roller experiences deflection deformation in the bearing direction. In the stress plane shown in the figure, when a sensor rotates to the upper part above the axis along with the flatness roller, the surrounding roller body is subjected to axial compressive stress, which expands the mounting hole and reduces the stress on the sensor. When the sensor is rotated to the bottom below the axis, the surrounding roller body is subjected to axial tensile stress, which causes the mounting hole to shrink and the force on the sensor to increase.

To further confirm the above conclusion, the theory of a simply supported deep beam in elastic mechanics was used to analyse the stress and strain of the detection roller. ${ }^{28)}$ As shown in Fig. 9, the roller is approximately regarded as a solid cylinder, ignoring its internal structural features. A uniformly distributed load $q$ is applied above the roller. The body length and diameter of the roller are $2 l$ and $D$, respectively. The two ends of the roller body are simple fulcrum points, one of which can slide. In the graphical coordinate system, the stress component at any position point $(x, y)$ is

$$
\left\{\begin{array}{l}
\sigma_{x}=\frac{6 q}{D^{3}}\left(l^{2}-x^{2}\right) y+q \frac{y}{D}\left(4 \frac{y^{2}}{D^{2}}-\frac{3}{5}\right) \\
\sigma_{y}=-\frac{q}{2}\left(1+\frac{y}{D}\right)\left(1-\frac{2 y}{D}\right)^{2} \\
\tau_{x y}=-\frac{6 q}{D^{3}} x\left(\frac{D^{2}}{4}-y^{2}\right)
\end{array}\right.
$$

where $\sigma_{x}$ is the normal stress in the $x$-direction; $\sigma_{y}$ is the normal stress in the $y$-direction; and $\tau_{x y}$ is the shear stress of the roller.

According to the stress-strain relationship, the strain components are

$$
\left\{\begin{array}{l}
\varepsilon_{x}=\frac{1}{E}\left(\sigma_{x}-\mu \sigma_{y}\right) \\
\varepsilon_{y}=\frac{1}{E}\left(\sigma_{y}-\mu \sigma_{x}\right) \\
\gamma_{x y}=\frac{2(1+\mu)}{E} \tau_{x y}
\end{array}\right.
$$

where $\varepsilon_{x}$ is the positive strain in the $x$-direction; $\varepsilon_{y}$ is the positive strain in the $y$-direction; $\gamma_{x y}$ is the shear strain; and $E$ and $\mu$ are the elastic modulus and Poisson's ratio of the roller.

The length of the roller body is $1500 \mathrm{~mm}$, the diameter

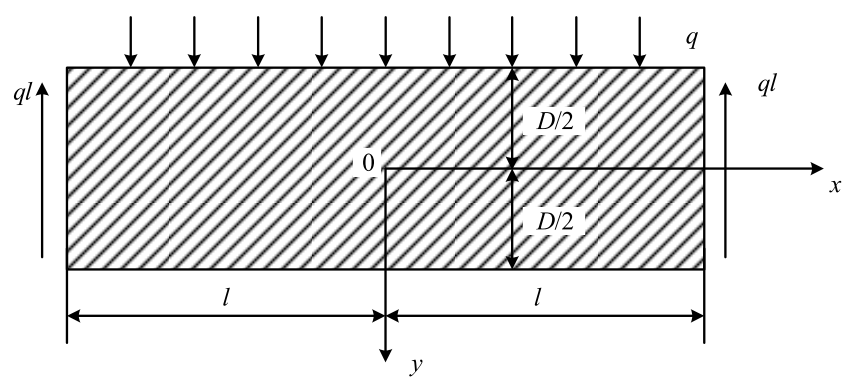

Fig. 9. Detection roller stress model.

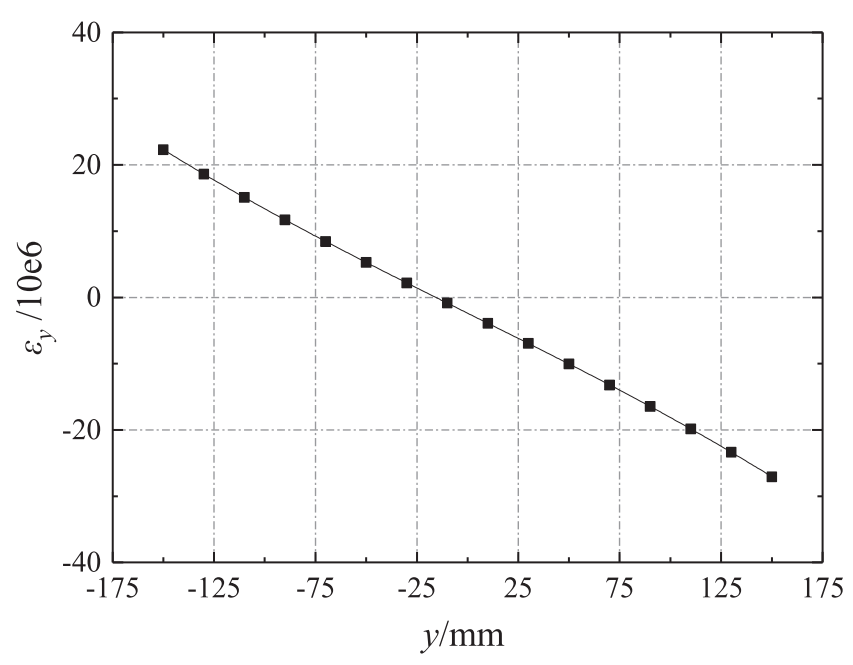

Fig. 10. Distribution of $\varepsilon_{y}$ at the symmetric cross section $(0, y)$ of the detection roller.

of the roller is $300 \mathrm{~mm}$, the elastic modulus is $2.1 \mathrm{e} 5 \mathrm{MPa}$, the Poisson's ratio is 0.3 and the distributed load is $q=1$ $\mathrm{MPa}$. The $y$-direction normal strain $\varepsilon_{y}$ distribution at the symmetric cross section $(0, y)$ of the roller is shown in Fig. 10. It can be seen from the figure that in the lower part of the roller $(y>0)$, especially at the roller surface, $\varepsilon_{y}$ is negative, resulting in compression deformation, compression of the mounting hole and increased force on the sensor. In the upper part of the roller $(y<0), \varepsilon_{y}$ is positive, resulting in tensile deformation, expansion of the mounting hole, and reduced stress on the sensor.

Under ideal conditions, the deflection waveform of the two sensors connected in parallel can be completely offset without considering the machining accuracy, sensor installation error, sensor sensitivity difference and other factors. However, it is impossible to guarantee such consistency in actual manufacturing installations. Therefore, the additional waveform cannot be completely eliminated by means of sensors parallel connection. In the rolling process, the bending degree of the roller body is increased due to the large tension loading, which results in the influence of the deflection waveform greatly increasing. If the peak value of the signal is directly extracted as the radial pressure on the detection unit to calculate the flatness distribution without considering the deflection influence, then a large detection error will arise. The additional signal varies with the variation in parameters such as the magnitude of the tension, the distribution of the tension, the width of the strip and the strip deviation. If the deflection of the roller under different working conditions is theoretically calculated and then the 
additional signal of each detection unit is further analysed, the difficulty and workload would be very large. Starting from the engineering application approach, as long as the corresponding deflection influence waveform can be found according to the actual waveform of each detection unit, the deflection waveform can be eliminated from the detected waveform, and the detection accuracy can be improved regardless of how the technological parameters change and how the deflection of the roller is distributed along its axis.

\section{Identification and Elimination of the Additional Deflection Waveform}

The effects of eliminating the deflection waveform by using the Fourier transform and digital filtering methods are shown in Fig. 11. Both methods can basically eliminate the deflection signal. However, the Fourier transform method is not suitable for online application because of its slow speed and large number of sampling points. The digital filtering method is suitable when the rolling speed, the additional signal and the effective signal frequency are fixed, but it is difficult to apply it to industrial flatness detection with strong time-varying characteristics at the rolling speed and the rotational speed of the detection roller.

To improve the precision of the online measurement of flatness in industrial applications, this paper presents a method to quickly identify and eliminate the additional signal of the integral roller flatness meter, which can not only solve the problem of the additional signal but also solve the zero drift problem.

Assuming that the sampling frequency of the flatness signal processor is $f$, the diameter of the roller is $D$ and the linear velocity of the roller is $v$, the number of sampling points obtained when the roller body rotates once is

$$
n=\frac{\pi D}{v} f
$$

The values corresponding to the sampling points in a rotation period are $y_{1}, y_{2}, \ldots, y_{i}, \ldots, y_{n}$.

Assume that the waveform curve of the additional signal is

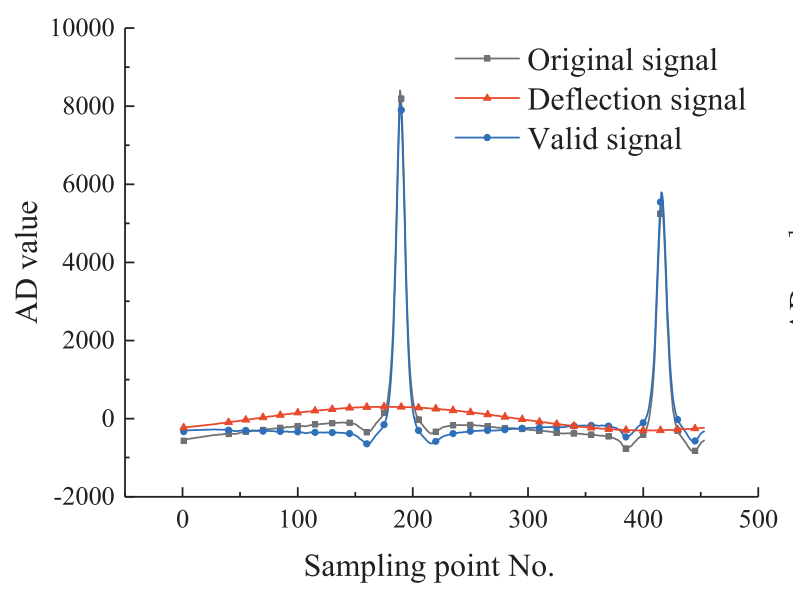

a) Fourier transform method

$$
y_{c}=a \sin \left(\frac{2 \pi i}{n}+\varphi\right)+b
$$

where $a$ is the amplitude, $\varphi$ is the initial phase and $b$ is the zero drift of the curve, as shown in Fig. 12.

Since the circumferential position of the sensor in the detection roller is fixed and thus the phase of the deflection influence waveform of each detection unit is fixed, the waveform peaks are also fixed in each detection unit. Suppose that the serial number of the signal peak point of sensor $\mathrm{A}$ is $i_{\mathrm{A}}$; then, that of sensor $\mathrm{B} i_{\mathrm{B}}$ is

$$
i_{\mathrm{B}}=i_{\mathrm{A}}+\lceil n / 2\rceil
$$

The initial phase of the waveform is

$$
\varphi=\frac{\pi}{2}-\frac{2 \pi i_{\mathrm{A}}}{n}
$$

Then, the waveform curve is

$$
y_{c}=a \sin \left[\frac{2 \pi}{n}\left(i-i_{\mathrm{A}}\right)+\frac{\pi}{2}\right]+b
$$

A method called the minimum error method is applied to divide the detected signal waveform and find the waveform range obviously superimposed with the deflection signal to extract the specific curve function of the deflection signal

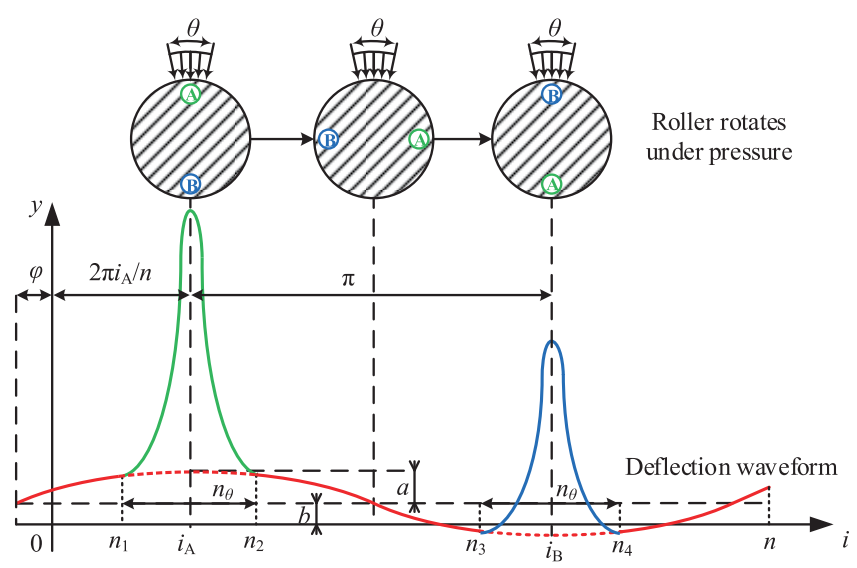

Fig. 12. Features of the additional signal waveform.

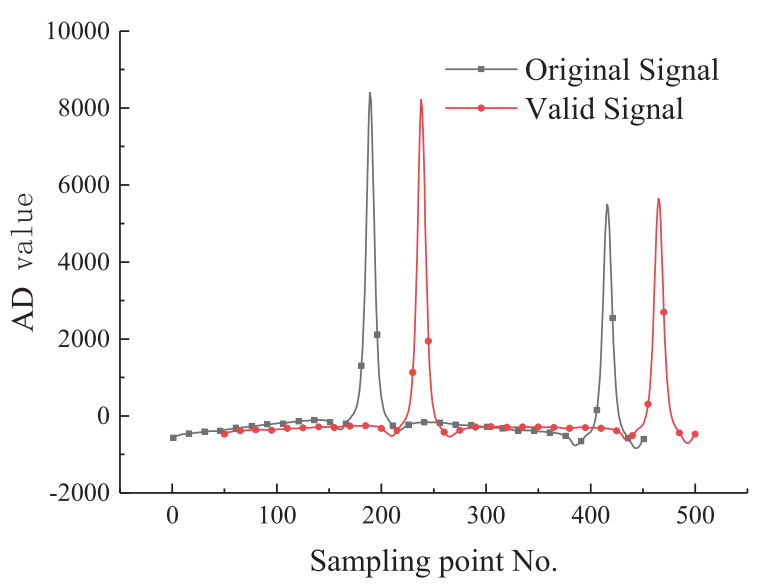

b) Digital filtering method

Fig. 11. Effects of Fourier transform and digital filtering methods. 
and finally remove it from the detected signal. The angle of the strip wrapping the detection roller is taken as the phase range of the peak section, and the corresponding sampling number is

$$
n_{\theta}=\frac{n \cdot \theta}{2 \pi}
$$

The number of sampling points that do not participate in the identification calculation of the deflection waveform within one rotation period is

$$
r=m \cdot n_{\theta}
$$

In the formula, $m$ is the number of sensors arranged circumferentially in each detection unit, that is, the number of peaks in a rotation period of the roller. $r=2 n_{\theta}$, as $m=2$ is taken here as an example.

The serial numbers of the sampling points at the two peak boundaries of the detection signal (that is, the sampling points not involved in the calculation of the deflection waveform identification) are

$$
n_{1}=i_{\mathrm{A}}-\frac{n_{\theta}}{2}, n_{2}=i_{\mathrm{A}}+\frac{n_{\theta}}{2}, n_{3}=i_{\mathrm{B}}-\frac{n_{\theta}}{2}, n_{4}=i_{\mathrm{B}}+\frac{n_{\theta}}{2} \ldots
$$

The error between the sampling point values and deflection waveform curve values is

$$
\begin{aligned}
& Q=\sum_{i=1}^{n}\left(y_{c}-y_{i}\right)^{2} \\
& =\sum_{i=1}^{n}\left\{a \sin \left[\frac{2 \pi}{n}\left(i-i_{\mathrm{A}}\right)+\frac{\pi}{2}\right]+b-y_{i}\right\}^{2},\left(i \neq n_{1} \sim n_{2}, n_{3} \sim n_{4}\right)
\end{aligned}
$$

According to the principle of minimum error, the governing equations are obtained as follows:

$$
\begin{aligned}
& \frac{\partial Q}{\partial a}=\sum_{i=1}^{n}\left\{a \sin \left[\frac{2 \pi}{n}\left(i-i_{\mathrm{A}}\right)+\frac{\pi}{2}\right]+b-y_{i}\right\} \sin \left[\frac{2 \pi}{n}\left(i-i_{\mathrm{A}}\right)+\frac{\pi}{2}\right] \\
& =\sum_{i=1}^{n}\left\{\begin{array}{l}
a \sin ^{2}\left[\frac{2 \pi}{n}\left(i-i_{\mathrm{A}}\right)+\frac{\pi}{2}\right]+b \sin \left[\frac{2 \pi}{n}\left(i-i_{\mathrm{A}}\right)+\frac{\pi}{2}\right] \\
-y_{i} \sin \left[\frac{2 \pi}{n}\left(i-i_{\mathrm{A}}\right)+\frac{\pi}{2}\right]
\end{array}\right\}=0 \\
& \frac{\partial Q}{\partial b}=\sum_{i=1}^{n}\left\{a \sin \left[\frac{2 \pi}{n}\left(i-i_{\mathrm{A}}\right)+\frac{\pi}{2}\right]+b-y_{i}\right\} \\
& =0,\left(i \neq n_{1} \sim n_{2}, n_{3} \sim n_{4}\right)
\end{aligned}
$$

The matrix form of the above equation is

$$
\begin{aligned}
& {\left[\begin{array}{cc}
\sum_{i=1}^{n}\left\{a \sin \left[\frac{2 \pi}{n}\left(i-i_{\mathrm{A}}\right)+\frac{\pi}{2}\right]\right\}^{2} & \sum_{i=1}^{n} a \sin \left[\frac{2 \pi}{n}\left(i-i_{\mathrm{A}}\right)+\frac{\pi}{2}\right] \\
\sum_{i=1}^{n} \sin \left[\frac{2 \pi}{n}\left(i-i_{\mathrm{A}}\right)+\frac{\pi}{2}\right] & n-r
\end{array}\right]\left[\begin{array}{l}
a \\
b
\end{array}\right]} \\
& =\left[\begin{array}{c}
\sum_{i=1}^{n} y_{i} \sin \left[\frac{2 \pi}{n}\left(i-i_{\mathrm{A}}\right)+\frac{\pi}{2}\right] \\
\sum_{i=1}^{n} y_{i}
\end{array}\right],\left(i \neq n_{1} \sim n_{2}, n_{3} \sim n_{4}\right)
\end{aligned}
$$

Then, the waveform amplitude $a$ and zero drift $b$ can be solved as follows:

$$
\begin{aligned}
{\left[\begin{array}{l}
a \\
b
\end{array}\right]=\left[\begin{array}{cc}
\sum_{i=1}^{n}\left\{\sin \left[\frac{2 \pi}{n}\left(i-i_{\mathrm{A}}\right)+\frac{\pi}{2}\right]\right. \\
\sum_{i=1}^{n} \sin \left[\frac{2 \pi}{n}\left(i-i_{\mathrm{A}}\right)+\frac{\pi}{2}\right] & \left.\sum_{i=1}^{n} \sin \left[\frac{2 \pi}{n}\left(i-i_{\mathrm{A}}\right)+\frac{\pi}{2}\right]\right]^{-1} \\
n-r
\end{array}\right] } \\
{\left[\begin{array}{c}
\sum_{i=1}^{n} y_{i} \sin \left[\frac{2 \pi}{n}\left(i-i_{\mathrm{A}}\right)+\frac{\pi}{2}\right] \\
\sum_{i=1}^{n} y_{i}
\end{array}\right],\left(i \neq n_{1} \sim n_{2}, n_{3} \sim n_{4}\right) }
\end{aligned}
$$

The additional signal waveform $y_{c}$ of the detection roller in the original signal $y_{i}$ is removed, and the real flatness signal $y_{e}$ is obtained as follows:

$$
y_{e}=y_{i}-a \sin \left[\frac{2 \pi}{n}\left(i-i_{\mathrm{A}}\right)+\frac{\pi}{2}\right]-b
$$

An identification and elimination of the additional waveform of a detection unit result obtained by using the above method is shown in Fig. 13, with amplitude $a=210$, phase $\varphi=-0.05 \pi$ and zero drift $b=-305$. The deflection waveform coincides almost completely with the original detected signal waveform for the phases outside the peak regions, and the valid signal waveform after elimination is very straight outside the peak regions, which proves the effectiveness of the method.

If the additional deflection signal of each detection unit is processed in real time and the influence of technological parameters is eliminated, then each detection unit will detect the actual force on the roller surface, so the detection accuracy of the flatness meter can improve. Take a 1500 $\mathrm{mm}$ six-high rolling mill integral roller flatness meter as an example. The rolled strip is made of SPCC and is 0.800 $\mathrm{mm}$ thick and $1000 \mathrm{~mm}$ wide with a $40 \mathrm{kN}$ outlet tension loaded. Figure 14 shows the comparison of the flatness detection results before and after elimination of the deflection signal. The radial pressure and the strip flatness distribution show obvious zigzag characteristics before elimination of the deflection signal. The overall flatness difference value is approximately $12.5 \mathrm{I}$, and the flatness fluctuation

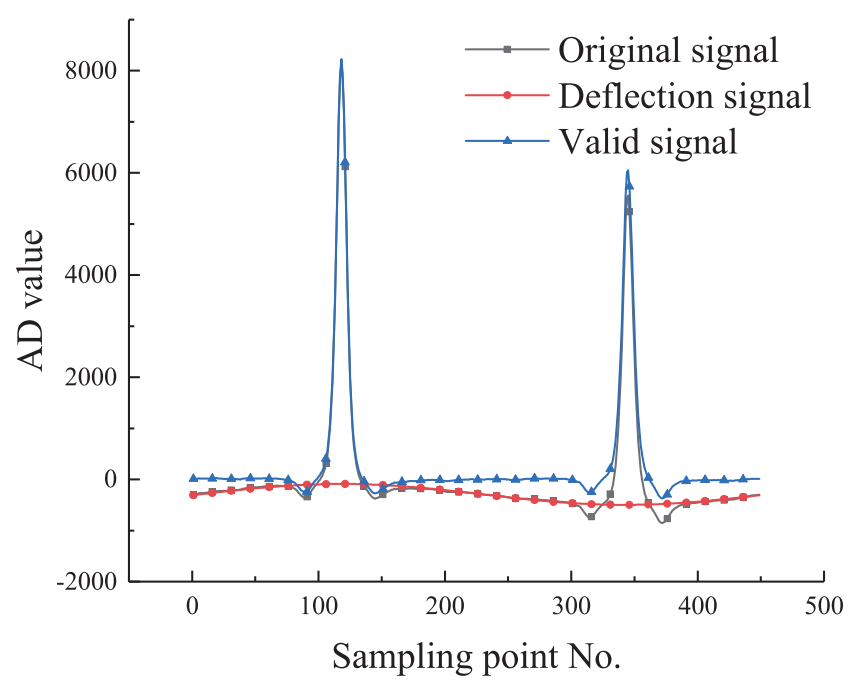

Fig. 13. Effect of the minimum error method. 


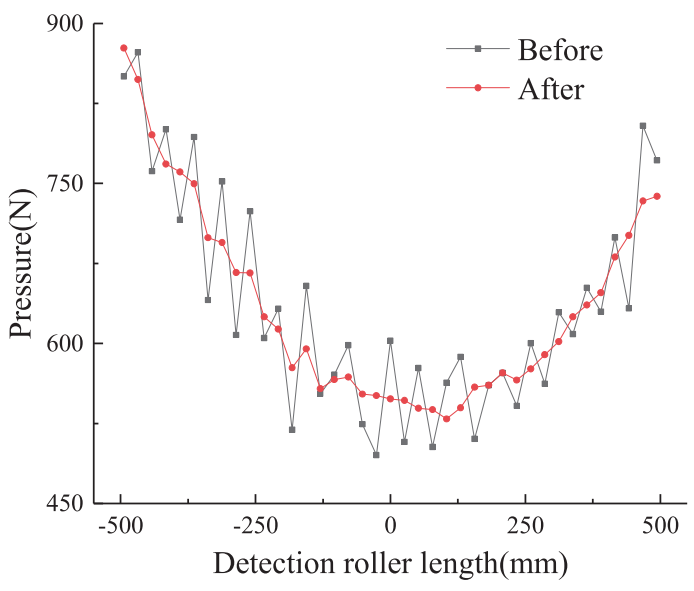

a) Pressure distribution

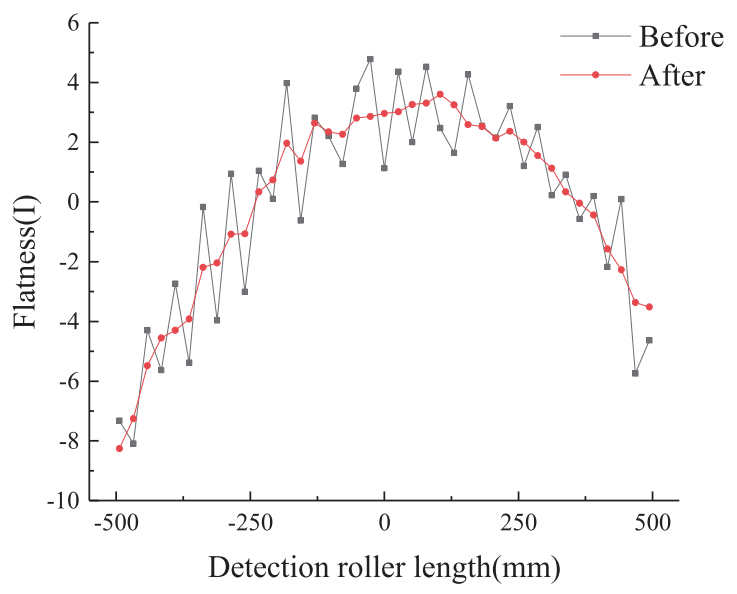

b) Flatness distribution

Fig. 14. Pressure and flatness distribution before and after removal of the deflection influence.

of adjacent detection units is up to 5 I. After eliminating the influence of deflection, the radial pressure and the strip flatness distribution are much smoother. The overall flatness difference becomes approximately $10 \mathrm{I}$, and the flatness fluctuation of adjacent units becomes less than $1.8 \mathrm{I}$, which is in accordance with the actual engineering situation.

The deflection signal also has a significant influence on the flatness control effect. A target curve of flatness control is generally required in the rolling process, which can be expressed as

$$
f(y)=c_{0}+c_{1}\left(\frac{2 y}{B}\right)+c_{2}\left(\frac{2 y}{B}\right)^{2}+c_{4}\left(\frac{2 y}{B}\right)^{4} \ldots
$$

where $y$ is the coordinate with the centre of the strip width as the origin; $B$ is the thickness of the strip; and $c_{0}, c_{1}, c_{2}$ and $c_{4}$ are the zeroth degree, first degree, second degree and fourth degree coefficients of the flatness control target curve, respectively.

During the flatness control process, the coefficients of the detected flatness distribution are subtracted from those of the target flatness distribution. Generally, the roller inclining is used to adjust the first degree flatness deviation, the roller bending is used to adjust the second degree flatness deviation, and the roller subsection cooling is used to adjust the fourth degree flatness deviation. The minimum error method is used to identify the flatness coefficients before and after elimination of the additional signals, and the results are compared with the target flatness coefficients, as shown in Table 1. It can be seen from the table that the coefficients of the terms after interference signal elimination are all improved. For example, the quadratic coefficient of the minimum error method after elimination of interference signals differs by 0.02 I from the target flatness coefficient, which has entered the control dead zone $( \pm 0.3 \mathrm{I})$. At this point, the current value of the roll bending force will remain unchanged until the difference between the two exceeds the dead zone before a new adjustment. However, the quadratic coefficient before the additional signal is eliminated differs by $0.61 \mathrm{I}$ from the target flatness coefficient, which is outside the dead zone. Therefore, the control system
Table 1. Comparison of flatness recognition coefficients before and after elimination.

\begin{tabular}{cccc}
\hline Flatness coefficients & Target & Before & After \\
\hline$a_{1}(\mathrm{I})$ & 0 & 1.60 & 1.34 \\
$a_{2}(\mathrm{I})$ & -6 & -6.61 & -5.98 \\
$a_{4}(\mathrm{I})$ & 0 & -1.39 & -0.38 \\
\hline
\end{tabular}

would consider that the roll bending force should be further increased at this time to reduce the difference between the two, which eventually would lead to the intermediate wave degree of the physical flatness exceeding the target value. In summary, by eliminating the additional deflection signal, not only the detection accuracy of the flatness meter but also the flatness control accuracy can be improved.

\section{Conclusion}

(1) The ideal output signal of an integral roller flatness meter sensor is a waveform similar to a pulse signal, whose peak value represents the radial pressure received by the roller surface of the corresponding detection unit. The output signal values outside the peak regions should be zero. When the roller surface is not subjected to any load, the signal values in the peak regions should also be zero.

(2) Under the action of the dead weight or an external load, the integral detection roller will experience deflection deformation, which will lead to a sinusoidal waveform with a $360^{\circ}$ full cycle distribution along the circumference. This waveform exists under no load and has a larger amplitude under loading. The upper part of the roller in the bearing plane is subjected to axial pressure, expanding the sensor mounting hole and reducing the force on the sensors and the output signal values. The lower part of the roller in the bearing plane is subjected to axial tension, shrinking the sensor mounting hole and increasing the force on the sensors and the output signal values. The waveform is an interference signal and should be identified and eliminated.

(3) Curve fitting through the minimum error method can accurately and quickly identify and eliminate the deflection 
signal. Not only the waveform but also the zero drift can be identified and eliminated with universality and generality. By using the method, the values outside the peak regions of the signal become approximately zero, as expected, and the calculation time is fast enough to meet the requirements of online application.

(4) If the deflection waveforms in all detection units are not eliminated, then the detected strip pressure and flatness distribution will exhibit an obvious zigzag shape, and the flatness value fluctuation of adjacent channels can reach 5 I. After eliminating the additional waveforms, the strip pressure and flatness distribution become smooth, and the flatness difference between adjacent channels is within $1.8 \mathrm{I}$, which conforms to the actual situation of the flatness value gradually changing along the width direction of the strip. After eliminating the influence of deflection, the flatness control precision can be improved by 2-3 I.

\section{REFERENCES}

1) S. Abdelkhalek, P. Montmitonnet, N. Legrand and P. Buessler: Int. J. Mech. Sci., 53 (2011), 661.

2) M. Jelali: J. Process Control, 17 (2007), 805

3) J. Molleda, R. Usamentiaga and D. F. García: Sensors, 13 (2013), 10245.

4) L. J. Chen, B. Han, W. Tan, A. P. Ruan and S. Z. Wang: Steel Roll., 29 (2012), 38 (in Chinese).

5) D. C. Wang, H. M. Liu and J. Liu: Chin. J. Mech. Eng., 30 (2017), 1248.

6) H. X. Yu, D. C. Wang, H. M. Liu, S. Zhang and L. P. Yang: Iron Steel, 54 (2019), 56 (in Chinese).

7) B. Q. Yu, L. P. Yang, H. M. Liu, Y. Peng and L. You: Chin. J. Sci. Instrum., 31 (2010), 904 (in Chinese).

8) L. P. Xu, L. You, D. H. Wang and X. T. Su: China Metall., 24 (2014),
23 (in Chinese).

9) X. G. Liang, Z. J. Jiao, G. D. Wang, X. H. Liu, J. F. Guo, H. T. Li and G. Wang: Metall. Equip., 6 (2006), 36 (in Chinese).

10) S. Abdelkhalek, P. Montmitonnet, M. Potier-Ferry, H. Zahrouni, N. Legrand and P. Buessler: Ironmaking Steelmaking, 37 (2010), 290.

11) D. C. Tran, N. Tardif and A. Limam: Int. J. Solids Struct., 69-70 (2015), 343.

12) B. Guan, Y. Zang, X. Han and K. Zheng: Sensors, 18 (2018), 1638.

13) D. Weisz-Patrault: Int. J. Solids Struct., 56-57 (2015), 175.

14) Y. Isei, T. Kato, M. Osugi and T. Ohta: Tetsu-to-Hagané, 105 (2019), 20 (in Japanese)

15) S. Aoe, M. Miyake and K. Kabeya: ISIJ Int., 57 (2017), 1054.

16) H. M. Liu, B. Q. Yu, L. P. Yang, Y. Peng, D. Ding, Z. M. Li and Q. Fu: Seamless Wireless Type Plate Shape Gauge of Entire Roller, China Patent CN101694368A, (2010) (in Chinese).

17) H. M. Liu, B. Q. Yu, L. P. Yang, D. Ding, B. Q. Yu and S. W. Chen: Compact Wireless Embedded Signal Processor Applied to ColdRolled Strip Shape Meter, China Patent CN105005287A, (2015) (in Chinese).

18) H. M. Liu, J. Liu, B. Q. Yu, L. P. Yang and Y. Zhang: J. Mech. Eng., 53 (2017), 87 (in Chinese).

19) H. X. Yu, D. C. Wang, H. M. Liu, T. Y. Zhang and L. P. Yang: ISIJ Int., 60 (2020), 939.

20) J. W. Liu, D. H. Zhang, J. S. Wang and P. F. Wang: J. Iron Steel Res. Int., 17 (2010), 35.

21) D. C. Wang, Y. H. Xu, H. X. Yu and H. M. Liu: Iron Steel, 55 (2020), 56 (in Chinese).

22) H. M. Wu, H. M. Liu, B. Q. Yu and L. P. Yang: J. Cent. South Univ., 21 (2014), 4503.

23) H. M. Wu, G. H. Cui, D. Zhang and H. M. Liu: Sensors, 18 (2018), 1988.

24) H. M. Wu, H. M. Liu and K. Wang: Int. J. Adv. Manuf. Technol., 94 (2018), 3189.

25) L. P. Yang, B. Q. Yu and H. X. Yu: J. Iron Steel Res. Int., 18 (2011), 28.

26) L. Yang, H. Yu, R. Li and W. Yuan: Ironmaking Steelmaking, 45 (2018), 457.

27) B. Q. Yu, Y. B. Sun, H. M. Liu, L. You and Y. Peng: J. Iron Steel Res. Int., 17 (2010), 21.

28) Z. L. Xu: Theory of Elasticity, Higher Education Press, Beijing, (2006), 49 (in Chinese). 